\title{
Core Electron-Root Confinement (CERC) in Helical Plasmas
}

M. Yokoyama 1), H. Maaßberg 2), C.D. Beidler 2), V. Tribaldos 3), K. Ida 1), T. Estrada 3), F. Castejon 3), A. Fujisawa 1), T. Minami 1), T. Shimozuma 1), Y. Takeiri 1), A.Dinklage 2), S. Murakami 4) and H. Yamada 1)

1) National Institute for Fusion Science, Toki 509-5292, Japan

2) Max-Planck Institut fur Plasmaphysik, Greifswald 17491, Germany

3) Laboratorio Nacional de Fusion, As. EURATOM-CIEMAT, Madrid 28040, Spain

4) Department of Nuclear Engineering, Kyoto Univ., Kyoto 606-8501, Japan

e-mail contact of main author: yokoyama@LHD.nifs.ac.jp

TEL: $+81-572-58-2278$

FAX: $+81-572-58-2630$

PACS: 52.25.Fi, 52.55.Hc 
Abstract. The improvement of core electron heat confinement has been realized in a wide range of helical devices such as CHS, LHD, TJ-II and W7-AS. Strongly peaked electron temperature profiles and large positive radial electric field, $E_{\mathrm{r}}$, in the core region are common features for this improved confinement. Such observations are consisitent with a transition to the "electron-root" solution of the ambipolarity condition for $E_{\mathrm{r}}$ in the context of the neoclassical transport, which is unique to non-axisymmetric configurations. Based on this background, this improved confinement has been collectively dubbed "core electron-root confinement" (CERC). The thresholds for CERC establishment are found for the collisionality and electron cyclotron heating (ECH) power. The magnetic configuration properties (e.g., effective ripple and magnetic islands/rational surfaces) play important roles for CERC establishment.

\section{Introduction}

Core electron-root confinement (CERC) is an improved confinement regime which is specific to helical systems. This regime has been obtained in quite different helical devices for ECH discharges above the power threshold depending on the magnetic configuration properties and heating scenarios [1,2]. It is based on a different mechanism than the internal transport barrier (ITB) in tokamaks, where the strongly sheared $\boldsymbol{E} \times \boldsymbol{B}$ flow within the narrow barrier screens off the turbulent transport; see e.g. the reviews $[3,4]$. In tokamaks, the ITB physics dominates both the particle and the ion energy balance whereas in helical devices CERC affects primarily the electron energy balance. A comparative study of CERC and ITB features was done for LHD and JT-60U [5].

According to neoclassical (NC) transport theory for the long-mean-free-path ( $\operatorname{lmfp}$ ) regime in helical plasmas, the electron-root $E_{\mathrm{r}}$ is of sufficient magnitude to limit the radial drift of ripple-trapped electrons, and thereby suppresses $1 / v$ transport [6,7]. Reducing this unfavorable $1 / v$ transport has been one of the major goals for stellarator optimization [8]. The tailoring of magnetic configurations (such as quasi-symmetric [9,10] and quasi-isodynamic [11] concepts) has been widely studied to achieve this. The suppression of $1 / v$ transport via the electron-root 
$E_{\mathrm{r}}$ makes another strategy possible and thus the understanding of CERC physics in the current and future helical devices for such an "electron-root perspective" is one of the main motivations of this international collaborative study.

The transitions between "ion-root" (with small magnitude of $E_{\mathrm{r}}$, usually negative) and the "electron-root" (with large positive $E_{\mathrm{r}}$ ) are based on a bifurcation mechanism. Such transitions become possible in the context of NC theory, when the ambipolarity condition of the $\mathrm{NC}$ fluxes, in which the transport coefficients depend on $E_{\mathrm{r}}$, allow several (an odd number) solutions. This feature specific to low-collisional helical plasmas leads to thresholds for the CERC establishment with respect to collisionality and $\mathrm{ECH}$ power depending on the magnetic configuration and the heating scenarios as well.

An activity to establish an International Stellarator Profile DataBase (ISPDB) has been initiated. The stellarator-specific CERC physics has been selected as the first topic of this collaboration, for which four helical devices, namely, CHS, LHD, TJ-II and W7-AS, can provide experimental results.

In Ref. [1], common features of CERC discharges, such as the large positive $E_{\mathrm{r}}$ and the improvement of the electron heat confinement, in these four devices are described. In this paper, effects of the ECH mode and the magnetic configuration property (effective ripple) on CERC establishment are the main topics, by which the difference of threshold value for the CERC appearance on different cases can be understood. In Sec. 2, NC transport theory in low-collisional helical plasmas is briefly reviewed which is relevant for the CERC interpretation. The characteristics of the magnetic field in four devices are described in Sec. 3, and CERC features will be documented in Sec. 4 with a focus on the effects of the magnetic configuration and heating scenario. Finally, summary and discussion are given in Sec. 5 .

\section{Neoclassical Transport Theory in Low-Collisional Helical Plasmas}


Helical devices produce their confining magnetic fields using external coils. Although this has obvious advantages such as steady-state and disruption-free operation, the three-dimensional nature of magnetic field produces localized particles with large $\mathrm{NC}$ transport rates (relative to axisymmetric tokamaks) at fusion-relevant plasma parameters. In the $\operatorname{lm} f p$ regime in helical plasmas, localized particles experience a uni-directional radial drift until pitch-angle collisions scatter them out of the local magnetic ripple in which they are trapped. This feature provides the so-called $1 / v$ regime with less frequent collisions leading to a larger transport. The radial excursion of the localized particles is limited, however, by the presence of $E_{\mathrm{r}}$, as the $\boldsymbol{E} \times \boldsymbol{B} \mathrm{drift}$ causes the localized particles to precess poloidally (following the helical trough in which they are trapped) with the frequency $\Omega_{\mathrm{E}}=E_{\mathrm{r}} / r B_{0}$. For the simplest picture of helical devices, the transport scales either as $v^{1 / 2}$ or $v$, depending on whether collisions or drifts are responsible for removing the particles from local ripples. It is possible to determine the mono-energetic diffusion coefficient, $D$, in each of these three $\operatorname{lm} f p$ regimes from asymptotic solutions of the kinetic equation and the scalings obtained may be summarized as $[6,12,13]$

$$
D_{1 / v} \propto \frac{\kappa^{2}}{v} \frac{\varepsilon_{\mathrm{eff}}^{3 / 2}}{R^{2}}, \quad D_{\sqrt{v}} \propto \frac{\kappa^{2} \sqrt{v}}{\left|\Omega_{\mathrm{E}}\right|^{3 / 2} R^{2}}, \quad D_{v} \propto\left(\frac{\kappa}{\Omega_{\mathrm{E}}}\right)^{2} \frac{v}{R^{1.5}},
$$

where $\varepsilon_{\text {eff }}$ is the so-called effective ripple for $1 / v$ transport, $\kappa$ the kinetic energy and $R$ the major radius. The $\varepsilon_{\text {eff }}$ condenses all effects of the magnetic topology on $1 / v$ transport into a single number and reflects the deviation from the symmetry of the magnetic configuration. These results, when combined with the ambipolarity constraint on the radial particle fluxes, $\Gamma_{\mathrm{e}}=\Gamma_{\mathrm{i}}$, lead to a non-linear equation which can have multiple solutions for $E_{\mathrm{r}}[14]$. This is a feature of $\operatorname{lmfp} \mathrm{NC}$ transport theory for helical devices which has no counterpart for axisymmetric tokamaks (where the transport coefficients are independent of $E_{\mathrm{r}}$ ).

Several features can be pointed out which are of relevance to the CERC discharges. Assuming that electrons are in the $1 / v$ regime and that $T_{\mathrm{e}} \sim T_{\mathrm{i}}$, the $E_{\mathrm{r}}$ must reduce $\Gamma_{\mathrm{i}}$ to the level of $\Gamma_{\mathrm{e}}$ and the electrons are therefore said to be the rate-controlling species. Often in this case, only a single solution for $E_{\mathrm{r}}$ exists, usually negative, which is refereed to as the ion root. This 
situation is recognized in Fig. 1(a) where $\mathrm{NC} \Gamma_{\mathrm{i}}$ and $\Gamma_{\mathrm{e}}$ (calculated by GSRAKE code [15]) are plotted as a function of $E_{\mathrm{r}}$ at a certain radius $(\rho=0.3)$ of an example non-CERC discharge in LHD. The ambipolarity provides ion-root $E_{\mathrm{r}}$. To a good approximation $v \propto \kappa^{-3 / 2}$ so that $1 / v$ transport coefficients scale as $T^{7 / 2}$. Any increase in $T_{\mathrm{e}}$ therefore leads to rapid growth in $\Gamma_{\mathrm{e}}$ (assuming other quantities to be unchanged) which the ions must follow. This process requires a reduction in the magnitude of $E_{\mathrm{r}}$ since even if $T_{\mathrm{i}}$ increases with $T_{\mathrm{e}}$ the transport coefficients in the $v^{1 / 2}$ or $v$ regimes scale only as $T^{5 / 4}$ and $T^{1 / 2}$, respectively. At some point, however, a second solution for $E_{\mathrm{r}}$ becomes possible (a third one appears simultaneously but is thermodynamically unstable) which is positive and of larger magnitude so as to suppress $1 / v$ transport of electrons. This solution is called the electron root and the ions are now the rate-controlling species. The strong reduction of both ion and electron transport coefficients leads to predictions of significantly improved NC transport when the electron root is realized. This situation is seen in Fig. 1(b) based on an example CERC discharge in LHD, where the electron-root $E_{\mathrm{r}}$ is predicted.

To obtain accurate values for NC transport coefficients in realistic helical devices, one must employ numerical methods which are capable of accounting for arbitrarily complex magnetic fields [16-19]. These several numerical methods based on different approach (Monte-Carlo, linearized drift-kinetic equation, bounce-averaged drift-kinetic equation, field-line-tracing method etc.) have been extensively benchmarked within an international collaboration and exhibit excellent agreement for the mono-energetic particle diffusion coefficients over a wide range of collision frequencies and values of $E_{\mathrm{r}}[20]^{1}$.

It should be emphasized that the NC theory used here is based on the assumption of local transport and is therefore incapable of describing effects of islands or stochastic regions on transport. The source-free treatment of the kinetic equation is also a limitation, particularly in the case of strong local heating of trapped electrons by X2-mode of ECH. Even for O1-mode heating of passing electrons, one must expect the high-energy portion of the distribution

${ }^{1}$ http://epsppd.epfl.ch/StPetersburg/pdf/P3_002.pdf 
function to deviate substantially from Maxwellian. For the discharges produced by strong $\mathrm{ECH}$ power described in this paper, one must therefore consider the NC values in this paper to represent a lower limit on the electron particle and heat fluxes.

\section{Description of the Four Helical Devices: CHS, LHD, TJ-II and W7-AS}

Both CHS ( 8 field periods) and LHD (10 periods) have magnetic configurations of the $l=2$ heliotron type [21]. The magnetic configurations used for CERC experiments are characterized by the position of the major radius, $R_{\mathrm{ax}}$, as: for CHS, $0.921<R_{\mathrm{ax}}<0.974 \mathrm{~m}$ and for LHD, $3.50<R_{\mathrm{ax}}<3.90 \mathrm{~m}$. Figures 2(a) and (b) show the mod- $B$ contours at the core region $(\rho=0.25)$ for CHS $\left(R_{\mathrm{ax}}=0.921 \mathrm{~m}\right)$ and $\operatorname{LHD}\left(R_{\mathrm{ax}}=3.75 \mathrm{~m}\right)$. Here the increment of $B$ in each contour is $1 \%$ of the magnitude. Due to the almost $\rho^{2}$-dependence of helical magnetic field amplitude produced by the $l=2$ helical coils, the $\bmod -B$ contours at the core region commonly follow almost the toroidal direction, which demonstrates that the $B$ varies in principle in the poloidal direction. The region of closed contours near the minimum- $B\left(B_{\min }\right)$ provides the localized particles and then $1 / v$ transport. Although the $\varepsilon_{\text {eff }}$ commonly increases towards the plasma periphery in heliotron configurations, it is rather small at the core region as easily recognized from Figs. 2(a) and (b).

TJ-II is a flexible heliac with 4 field periods where the bean-shaped flux surfaces rotate around the central conductor, composed of a circular and a helical coil. The ratio of the currents in the central conductors allows large variation of the rotational transform $(\imath)$, the plasma position, shape, and size to some extent [22]. The rotational transform profile is fairly flat (low shear). The mod- $B$ contours at $\rho=0.25$ is shown in Fig. 2(c), from which it is recognized that the helicity (almost $\rho$-dependence in a heliac) and the mirror magnetic field (almost independent on $\rho$ ) contributions are also remarkable in addition to the toroidicity (poloidal variation of $B$ ). The double-periodic (in the toroidal direction) magnetic field component also contributes to make two locations of $B_{\max }$ and $B_{\min }$. 
The magnetic configuration of W7-AS is partly drift optimized because of an average elongation of $\sim 2$. This averaged elongation reduces both the Shafranov shift and the NC transport in the Pfirsch-Schlüter as well as in the plateau regime, but not in the $\operatorname{lmfp}$ regime where localized ripples dominate. With increasing the rotational transform (and outward shift), the localized minima of $B$ deepens, in particular, at outer radii, and dominate the NC transport in $\operatorname{lm} f p$ regime. In the CERC discharges, mainly low- $\iota$ configurations were employed. In addition to the inward shift, a separated coil current supply allows for an independent variation of the toroidal mirror component controlling the power absorption for trapped electrons for ECH X2-mode. The standard configuration with $\imath(0) / 2 \pi \sim 1 / 3$ has a pronounced minimum of $B$ in the ECH launching plane. This $B_{\min }$ region is well described in Fig. 2(d) at the half-way of the toroidal period. The $B$ varies mainly in the toroidal direction, which indicates that the mirror magnetic field is predominant in W7-AS. This feature is not limited only in the core region, but also the case in the peripheral region.

The $\varepsilon_{\text {eff }}$ in the core region (where CERC is established) in the four devices are summarized here. The $\varepsilon_{\text {eff }}$ is the convenient parameter to compare different magnetic configurations (e.g., see Fig. 2) from the view point of $1 / v$ transport. In LHD, $\varepsilon_{\text {eff }}$ at the CERC region takes the smallest value at $R_{\mathrm{ax}}=3.75 \mathrm{~m}$ (close to zero), and both inward and outward shift of $R_{\mathrm{ax}}$ increase $\varepsilon_{\text {eff }}$ due to the appearance of the toroidal mirror component [23]. TJ-II takes the largest value of $\varepsilon_{\text {eff }}$ at the core region (but at the most only 0.03 ) among the four devices, and thus, it is expected to have the easiest access to the electron root. In such a sense, TJ-II can provide the unique experimental stage in this collaborative study. It should be noted here that TJ-II primarily aimed to enhance the magnetic well for MHD stability issues rather than aiming to reduce the NC transport. In W7-AS, $\varepsilon_{\text {eff }}$ is moderate in the core region $\left[\varepsilon_{\text {eff }}(0)<0.015\right]$ and increases only slowly with radius compared to the other configurations. The low-mirror configuration realized by the corner-coil current control has somewhat smaller $\varepsilon_{\text {eff }}$ in the core region.

\section{CERC Descriptions}


Figure 3 shows examples for $T_{\mathrm{e}}$ profiles (smoothed) in CERC discharges in the four devices. Within the CERC region, $T_{\mathrm{e}}$ is highly peaked. This feature is found at quite different $n_{\mathrm{e}}, P_{\mathrm{ECH}}$ (ECH power), and $B$, for example, as for the range of $n_{\mathrm{e}}, n_{\mathrm{e}} \sim 0.15 \times 10^{19} \mathrm{~m}^{-3}$ (at LHD) to $n_{\mathrm{e}} \sim 5.3 \times 10^{19} \mathrm{~m}^{-3}$ (at W7-AS) and the range of $P_{\mathrm{ECH}}, P_{\mathrm{ECH}} \sim 0.3 \mathrm{MW}$ (at TJ-II) to $n_{\mathrm{e}} \sim 1.2 \mathrm{MW}$ (at W7-AS). With increasing $n_{\mathrm{e}}$ at fixed $P_{\mathrm{ECH}}$, however, the $T_{\mathrm{e}}$-profile peaking becomes less pronounced. Figure 4 documents the CERC establishment through the increase of $P_{\mathrm{ECH}}$ at LHD [24] $\left(n_{\mathrm{e}} \sim 0.3 \times 10^{19} \mathrm{~m}^{-3}, B \sim 1.5 \mathrm{~T}, R_{\mathrm{ax}}=3.8 \mathrm{~m}\right)$. The power threshold for CERC establishment at these conditions was found to exceed $P_{\mathrm{ECH}}>0.58 \mathrm{MW}$. The appearance of the central $T_{\mathrm{e}}$-peaking (Fig. 4(a)) with the ECH power beyond the threshold value corresponds to the establishment of the electron-root $E_{\mathrm{r}}$ (Fig. 4(b)).

Figure 5 shows results from an ECH power scan experiment at LHD [25] $\left(R_{\mathrm{ax}}=3.75 \mathrm{~m}\right.$, $B=1.52 \mathrm{~T})$, where the normalized scale length of the $T_{\mathrm{e}}$ gradient, $R / L_{\mathrm{Te}}(R$ is the major radius), is shown as a function of $P_{\mathrm{ECH}} / n_{\mathrm{e}}$. It shows a clear jump at the threshold at $P_{\mathrm{ECH}} / n_{\mathrm{e}} \sim 1.4 \times 10^{19} \mathrm{MWm}^{3}$. This finding indicates that the CERC establishment is based on a bifurcation mechanism. On the contrary, no clear ECH power threshold exists in tokamak electron-ITB formation [5], which reflects that the CERC establishment is based on the bifurcation of $E_{\mathrm{r}}$ to the electron root, that is, the stellarator-specific physics. The ECH power threshold is found to increase with $n_{\mathrm{e}}$ [26-28]. Consequently, the $n_{\mathrm{e}}$ threshold for fixed $P_{\mathrm{ECH}}$ is also demonstrated at LHD [28], TJ-II [29] and W7-AS [30].

The effect of the magnetic configuration properties, in particular, of $\varepsilon_{\mathrm{eff}}$ as well as of the ECH power absorption by ripple-trapped electrons in the CERC region, has been analyzed in LHD and W7-AS. It is expected that CERC is more easily established in configurations with larger $\varepsilon_{\text {eff }}$ due to its easier access to the electron-root regime as predicted by NC transport theory. This was experimentally verified at the peripheral region in LHD [31]. Thus, the threshold for the CERC establishment with respect to $P_{\mathrm{ECH}}\left(n_{\mathrm{e}}\right)$ is expected to become lower (higher) in configurations with larger $\varepsilon_{\text {eff. }}$ 
This expectation regarding the CERC accessibility is experimentally verified by the O1-mode ECH experiments in W7-AS [32], as shown in Fig. 6. The ECH in O1-mode is nearly completely absorbed by passing electrons, and the effect of $\varepsilon_{\text {eff }}$ on the CERC establishment could be analyzed, since there is little contribution of the non-diffusive ECH-driven electron flux due to the heated ripple-trapped electrons. The CERC feature was clearly obtained in a configuration with larger $\varepsilon_{\mathrm{eff}}(0) \sim 0.011$ (solid curve in Fig. 6) for the same level of $P_{\mathrm{ECH}}$ and $n_{\mathrm{e}}$ compared to the lower $\varepsilon_{\text {eff }}(0) \sim 0.008$ configuration (dotted curve in Fig. 6). For the X2-mode $\mathrm{ECH}$, both $P_{\mathrm{ECH}}$ and $n_{\mathrm{e}}$ scans were performed [30] for two magnetic configurations in W7-AS: with a minimum of $B$ ("standard" configuration) and with a maximum of $B$ ("low-mirror" configuration) in the ECH launching plane. The $P_{\mathrm{ECH}}$ scan was conducted at fixed density of $n_{\mathrm{e}} \sim 2 \times 10^{19} \mathrm{~m}^{-3}$. While the CERC feature appears except for the lowest $P_{\mathrm{ECH}}$ (0.2 MW) for the "standard" configuration, it appears only at the highest $P_{\mathrm{ECH}}(1.2 \mathrm{MW})$ in the "low-mirror" configuration. Monte-Carlo simulations (GNET code [33]) for estimating the effect of ECH power absorbed in ripple-trapped electrons have shown that the ECH-driven electron flux is of comparable magnitude with the ambipolar NC electron flux in the ion-root regime, and is also larger in the "standard" configuration than in the "low-mirror" configuration. This numerical result was experimentally confirmed by the faster $T_{\mathrm{e}}$ decay after the switching-off of ECH [30]. Thus the ECH-driven electron flux also affects the accessibility to CERC establishment for the X2-mode heating scenario through the modification of ambipolar condition in addition to NC contributions.

The $P_{\mathrm{ECH}}$ scan was also conducted in different magnetic configurations in LHD. One of such results were already shown in Fig. 5, and the other results are shown in Fig. 7 which were obtained in a configuration with $R_{\mathrm{ax}}=3.5 \mathrm{~m}$ and $B=2.854 \mathrm{~T}$. The threshold value of $P_{\mathrm{ECH}} / n_{\mathrm{e}}$ for CERC establishment for the latter case is about the half of that for the former case (e.g., $0.7 \times 10^{19} \mathrm{MWm}^{3}$ in Fig. 7 vs. $1.4 \times 10^{19} \mathrm{MWm}^{3}$ in Fig. 5). The experimental conditions in these two cases are different in several points, which might provide a clue to understand this difference of threshold in $P_{\mathrm{ECH}} / n_{\mathrm{e}}$. One of the expected differences is the contribution of 
heated ripple-trapped electrons. The ECH heating mode is $\mathrm{X} 2(\mathrm{O} 1)$ for the former [Fig. 5] (latter [Fig. 7]) case, which indicates that the former case is expected to be easier for the CERC establishment from the viewpoint of the presence of heated ripple-trapped electrons. The threshold difference, however, can not be understood by this, which might be due to the rather small magnetic ripple in the core region of the LHD with less trapped particle fraction. This consideration is supported by the GNET calculation for an LHD-CERC discharge, in which ripple-trapped electron flux is at the most of $30 \%$ of the electron NC flux in a CERC discharge established by X2 mode ECH heating [24]. The other difference is $B$ itself. In Ref. [34], it is shown that the lower $B$, the lower the threshold for $T_{\mathrm{e}}$ to enter the electron root regime. This tendency, however, is also contradicting to understand the threshold difference. Then, reflecting to the argument for $\boldsymbol{\varepsilon}_{\mathrm{eff}}$ in LHD (see. Sec. 3), $\boldsymbol{\varepsilon}_{\mathrm{eff}}$ in the core region is larger in $R_{\mathrm{ax}}=3.5 \mathrm{~m}\left(\varepsilon_{\mathrm{eff}}(\rho=0.15) \sim 0.009\right.$ and almost unchanged towards the magnetic axis) than that in $R_{\mathrm{ax}}=3.75 \mathrm{~m} \quad\left(\varepsilon_{\mathrm{eff}}(\rho=0.15) \sim 0.004\right.$ and decreasing towards the axis $)$. This fact might be a plausible candidate to understand the lower $P_{\mathrm{ECH}} / n_{\mathrm{e}}$ in Fig. 7 compared to that in Fig. 5, as similar as in Fig. 6 (e.g., the larger $\boldsymbol{\varepsilon}_{\text {eff }}$, the easier the CERC is established).

Large positive $E_{\mathrm{r}}$ (electron root) in the CERC region is commonly observed in four devices. These findings are consistent with the picture of the NC ambipolarity (see. Sec. 2). Figure 8 shows example of the measured $E_{\mathrm{r}}$ (by the charge exchange recombination spectroscopy, CXRS) values for a CERC discharge in W7-AS in comparison to the NC ambipolar $E_{\mathrm{r}}$ calculated by the DKES code [35] with the measured density and temperature profiles. Several roots are found from the ambipolarity condition in this local approach, and the transition between the ion root and electron root (solid curve in Fig. 8) is obtained from the diffusion equation for $E_{\mathrm{r}}$ [36]. The bifurcation nature of the ambipolar $E_{\mathrm{r}}$ is confirmed in other devices as well (cf., Fig. 4(b) for LHD) to be the common physical mechanism for the CERC establishment.

The electron energy balance has also been analyzed for CERC discharges and compared with the NC prediction. The traditional picture has been employed here, where a purely diffusive 
ansatz for the electron heat flux density, $Q_{\mathrm{e}}=-n_{\mathrm{e}} \chi_{\mathrm{e}} T_{\mathrm{e}}$, , is used for the experimental power balance, and $\chi_{\mathrm{e}}(r)$ (the experimental electron heat diffusivity) is compared with the $\mathrm{NC}$ heat diffusivity. Figure 9 shows the comparison of the experimental $\chi_{\mathrm{e}}$ (error bars are estimated by least-squares fitting of the power balance) with the NC heat diffusivity in the same discharge as in Fig. 8. The NC $\chi_{\mathrm{e}}$ curve shown for the assumption $E_{\mathrm{r}}=0$ is provided as a theoretical "extension" of the expected electron heat diffusivity under ion root (small $E_{\mathrm{r}}$ ) assumptions. The experimental $\chi_{\mathrm{e}}$ is far below this curve. The NC bifurcation picture is also confirmed in spontaneous transitions in TJ-II where the central electrostatic potential (measured by Heavy Ion Beam Probe, HIBP) changes in an equivalent manner to the central $T_{\mathrm{e}}$ (measured by Electron Cyclotron Emission) and anti-correlated to the central $n_{\mathrm{e}}$ (proportional to the HIBP intensity) [37].

The impact of magnetic islands on the CERC establishment has also been recognized both at LHD $[27,38]$ and TJ-II $[39,40]$. The low-order $(z / 2 \pi=1 / 2)$ rational surface is located at the central region in LHD. The NBI-beam driven current can change its location and even make it disappear (in the case of the co-injection) with the modification of rotational transform value/profile. The clear threshold for the ECH power can be observed in the case of the counter-injection. On the other hand, it becomes unclear for the case of co-injection. This difference appears to be related to the presence of the $z / 2 \pi=1 / 2$ surface in the core region. In TJ-II, high configuration flexibility has been utilized to investigate the impact of low-order rational surfaces. A small inductive current is applied to vary the core rotational transform profile, which demonstrates the reduction of ECH power threshold for the CERC establishment when the $z / 2 \pi=3 / 2$ island is present in the central part of the discharge [37]. Systematic experiments with different low-order rationals have exhibited a dependence of the threshold density on the order of the rational surface (island width) [40]. The theoretical investigation for such experimental findings related to magnetic islands/rational surfaces are the future subject.

\section{Conclusion and Discussions}


Core electron-root confinement (CERC) has been commonly found in the four helical devices, CHS, LHD, TJ-II and W7-AS. CERC is clearly identified as an improved electron energy confinement related to the transition to the electron-root solution of the ambipolarity condition, as predicted by NC transport theory in low-collisional helical plasmas. Configurations with a large effective ripple in the core region allow for easier access to CERC. For ECH in X2-mode with significant absorption by ripple-trapped electrons, additional ECH-driven flux can reduce the ECH power threshold for CERC establishment, as was found in the configuration scan experiment in W7-AS. The impact of magnetic islands/rational surfaces on CERC establishment has also been recognized in both LHD and TJ-II experiments.

Some remarks on the CERC accessibility in the future devices are tried here based on the context of this paper. For quasi-axisymmetric configurations, like NCSX [41], the ECH power threshold is expected to increase due to its rather low $\varepsilon_{\text {eff. }}$ On the contrary, quasi-isodynamic configurations allow for a moderate $\varepsilon_{\text {eff }}$ at the core region due to the toroidal mirror field. For example, the configurational flexibility of W7-X [8] allows for a variation of the toroidal mirror field, resulting in the variation of $\varepsilon_{\text {eff }}(0)$. Together with the significant ECH power, CERC will probably be possible at higher densities in $\mathrm{W} 7-\mathrm{X}$ than in the experiments described here.

\section{Acknowledgements}

This work is supported in part by the grant from the Japanese Ministry of Education, Culture, Sports, Science, and Technology and in part by the NIFS Collaborative Research Program, NIFS04KLDB001. 


\section{References}

[1] YOKOYAMA, M., et al., Fusion Sci. Tech. 50 (2006) 327.

[2] FUJISAWA, A., et al., Plasma Phys. Control. Fusion 45 (2003) R1.

[3] WOLF, R.C., Plasma Phys. Contr. Fusion 45 (2003) R1.

[4] CONNOR, J.W., et al., Nucl. Fusion 44 (2004) R1.

[5] K. IDA et al., Plasma Phys. Control. Fusion 46, A45 (2004).

[6] GALEEV, A.A., and SAGDEEV, R.Z., Review of Plasma Physics 7 (1977) 307.

[7] KOVRIZHNYKH, L.M., Nucl. Fusion 24 (1984) 851.

[8] GRIEGER, G., et al., 13th International Conference Plasma Physics Controlled Nuclear Fusion Research, Washington, 1990 (IAEA, Vienna, 1991), Vol.3, p.525.

[9] NÜHRENBERG, J., and ZILL, R., Phys. Lett. A 129 (1988) 113.

[10] NÜHRENBERG, J., et al., Theory of Fusion Plasmas (Varenna 1994), Editrice Compositori, Bologna (1994), p.3.

[11]GORI, S., et al., "Quasi-isodynamic Stellarators," Theory of Fusion Plasmas (Varenna 1996), Editrice Compositori, Bologna (1996), p.335.

[12] BEIDLER, C.D., and HITCHON, W.N.G., Plasma Phys. Control. Fusion 36 (1994) 317.

[13] CRUME, E.C., Jr., et al., Phys. Fluids 31 (1988) 11.

[14] MYNICK, H.E., and HITCHON, W.N.G., Nucl. Fusion 23 (1983) 1053.

[15] BEIDLER, C.D., and D'HAESELEER, W.D., Plasma Phys. Control. Fusion 37 (1995)

463.

[16] van RIJ, W.I., and HIRSHMAN, S.P., Phys. Fluids B 1 (1989) 563.

[17] TRIBALDOS, V., Phys. Plasmas 8 (2001) 1229.

[18] MURAKAMI, S., et al., Nucl. Fusion 42 (2002) L19.

[19] NEMOV, V.V., et al., Phys. Plasmas 6 (1999) 4622.

[20] BEIDLER, C.D., et al., "NEOCLASSICAL TRANSPORT IN STELLARATORS

-RESULTS FROM AN INTERNATIONAL COLLABORATION", Proc. 30th EPS

Conference on Contr. Fusion and Plasma Phys., St. Petersburg, 7-11 July 2003, ECA Vol. 27A, (2003) P-3.2.

[21] UO, K., J. Phys. Soc. Japan 16 (1961) 1380.

[22] TRIBALDOS, V., and Van MILLIGEN, B.P., Nucl. Fusion 36 (1996) 283.

[23] YOKOYAMA, M., and WATANABE., K.Y., Nucl. Fusion 45 (2005) 1600.

[24] IDA, K., et al., Phys. Rev. Lett. 91 (2003) 085003.

[25] IDA, K., et al., Phys. Plasmas 11 (2004) 2551.

[26] TAKEIRI, Y., et al., Phys. Plasmas 10 (2003) 1788.

[27] TAKEIRI, Y., et al., Fusion Sci. Techn. 46 (2004) 106.

[28] SHIMOZUMA, T., et al., Plasma Phys. Control. Fusion 45 (2003) 1183.

[29] CASTEJON, F., et al., Nucl. Fusion 42 (2002) 271.

[30] MAAßBERG, H., et al., Phys. Plasmas 7 (2000) 295.

[31] YOKOYAMA, M., et al., J. Plasma Fusion Res. SERIES 6 (2004) 218.

[32] ROMÉ, M., et al., Plasma Phys. Control. Fusion 48 (2006) 353.

[33] MURAKAMI, S., et al., Nucl. Fusion 40 (2000) 693.

[34] YOKOYAMA, M., et al., Nucl. Fusion 42 (2002) 143.

[35] HIRSHMAN, S.P., et al., Phys. Fluids 29 (1986) 2951.

[36] MAAßBERG, H., et al., Phys. Fluids B 5 (1993) 3627.

[37] ESTRADA, T., et al., Plasma Phys. Control. Fusion 46 (2004) 277.

[38] SHIMOZUMA, T., et al., Nucl. Fusion 45 (2005) 1396.

[39] CASTEJON, F., et al., Nucl. Fusion 44 (2004) 593.

[40] ESTRADA, T., et al., Fusion Sci. Tech. 50 (2006) 127.

[41] NEILSON, G.H., et al., Phys. Plasmas 7 (2000) 1911. 


\section{Figure Captions}

Fig.1 Neoclassical ion $\left(\Gamma_{\mathrm{i}}\right)$ and and electron $\left(\Gamma_{\mathrm{e}}\right)$ fluxes as a function of $E_{\mathrm{r}}$ at $\rho=0.3$ of example (a) non-CERC and (b) CERC discharges in LHD. The $\Gamma_{\mathrm{r}}=\Gamma_{\mathrm{e}}$ provides ambipolar $E_{\mathrm{r}}$.

Fig. 2 Mod- $B$ contours at $\rho=0.25$ for (a) $\operatorname{CHS}\left(R_{\mathrm{ax}}=0.921 \mathrm{~m}\right)$, (b) LHD $\left(R_{\mathrm{ax}}=3.75 \mathrm{~m}\right)$, (c) TJ-II and (d) W7-AS (standard with low- $\iota(0) / 2 \pi)$. The increment of each iso-line is $1 \%$.

Fig.3 $T_{\mathrm{e}}$ profiles for example CERC discharges in the four devices.

Fig.4 (a) $T_{\mathrm{e}}$ and (b) $E_{\mathrm{r}}$ profiles measured at LHD with $P_{\mathrm{ECH}}=0,0.58$, and $0.78 \mathrm{MW}$ (from low to high $\left.T_{\mathrm{e}}(0)\right)$ on an NBI sustained plasma.

Fig.5 Normalized $T_{\mathrm{e}}$ gradient as a function of normalized ECH power (X2-mode) for CERC establishment in LHD $\left(R_{\mathrm{ax}}=3.75 \mathrm{~m}, B=1.52 \mathrm{~T}\right)$

Fig.6 CERC- $T_{\text {e }}$ profiles obtained in W7-AS (O1-mode ECH). The dotted (solid) curve is for $P_{\mathrm{ECH}}=0.69(0.65) \mathrm{MW}$ at $n_{\mathrm{e}}(0) \sim 4(5) \times 10^{19} \mathrm{~m}^{-3}$ in configurations with $\varepsilon_{\mathrm{eff}}(0) \sim 0.008(0.011)$.

Fig.7 Central $T_{\mathrm{e}}$ as a function of normalized ECH power (O1-mode) for CERC establishment in LHD $\left(R_{\mathrm{ax}}=3.5 \mathrm{~m}, B=2.854 \mathrm{~T}\right)$ for two different values of $n_{\mathrm{e}}$.

Fig.8 Measured $E_{\mathrm{r}}$ values with the NC ambipolar $E_{\mathrm{r}}$ in a CERC discharge in W7-AS.

Fig.9 Comparison between the experimental (exp.) and $\mathrm{NC} \chi_{\mathrm{e}}$ with and without considering the ambipolar $E_{\mathrm{r}}$ for the same CERC discharge as in Fig.8. 
Fig.1 NF/241006/PAP Yokoyama et al.
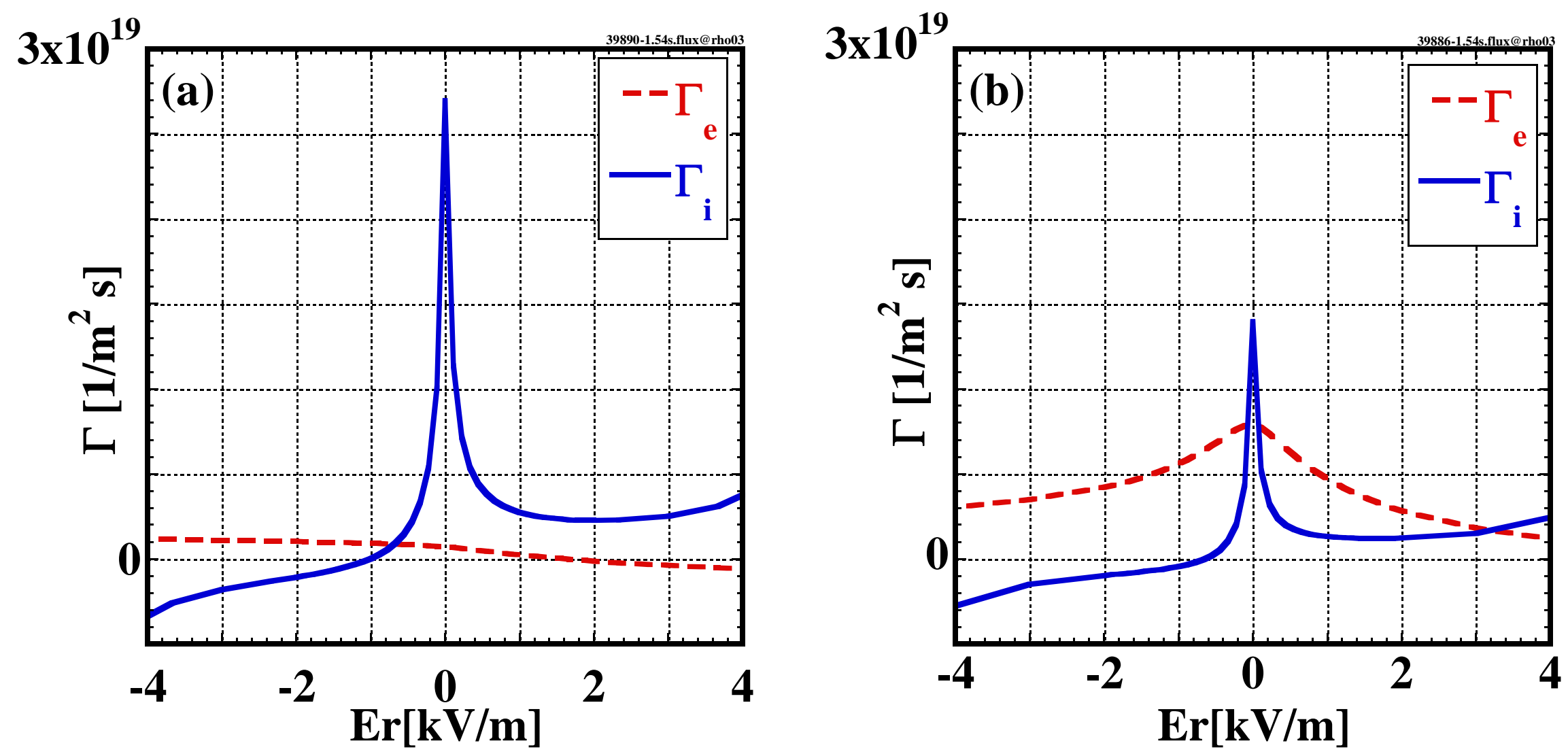

(Non-color on printed journal) 
Fig.2 NF/241006/PAP Yokoyama et al.
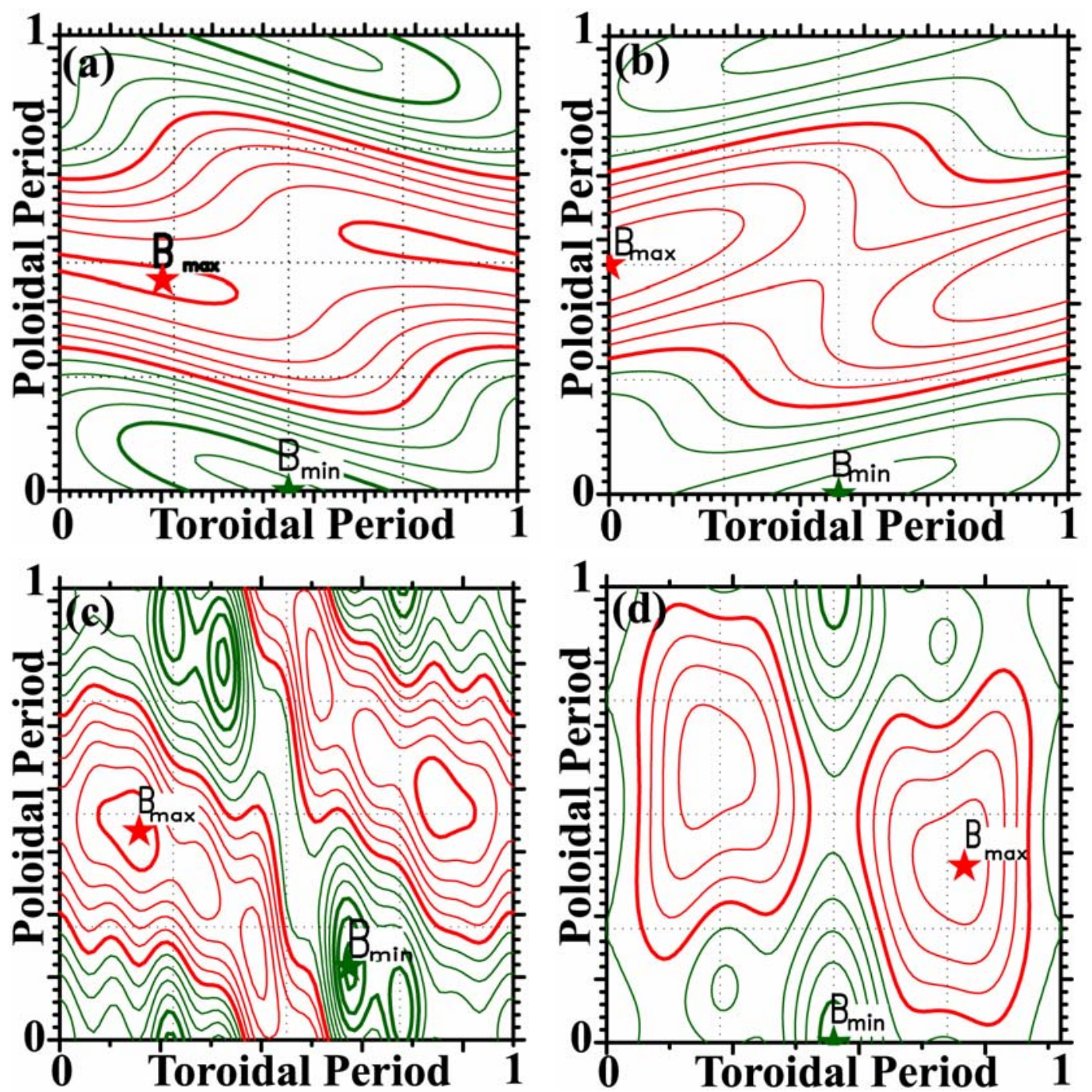

(Non-color on printed journal) 
Fig.3 NF/241006/PAP Yokoyama et al.

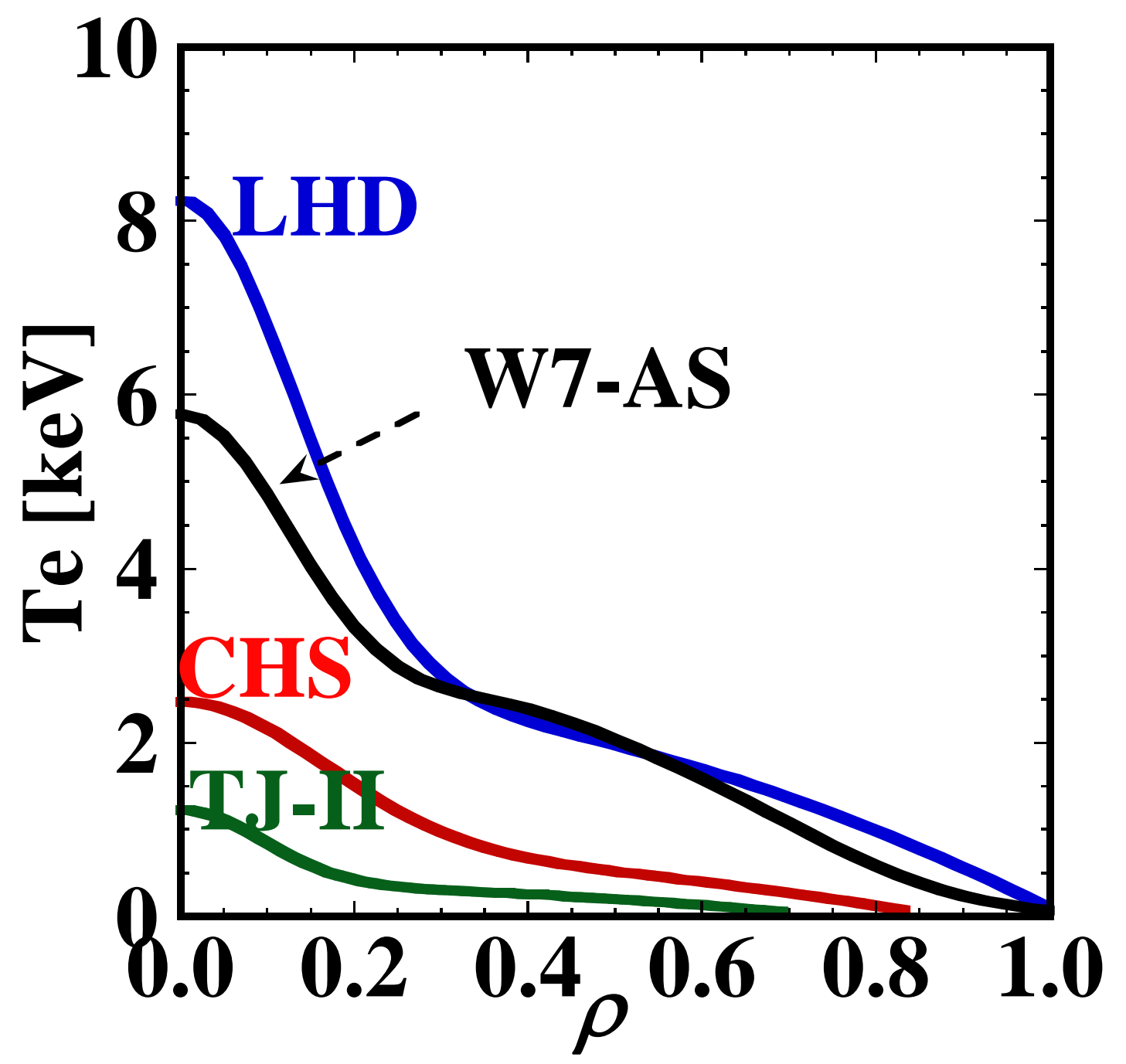

(Non-color on printed journal) 


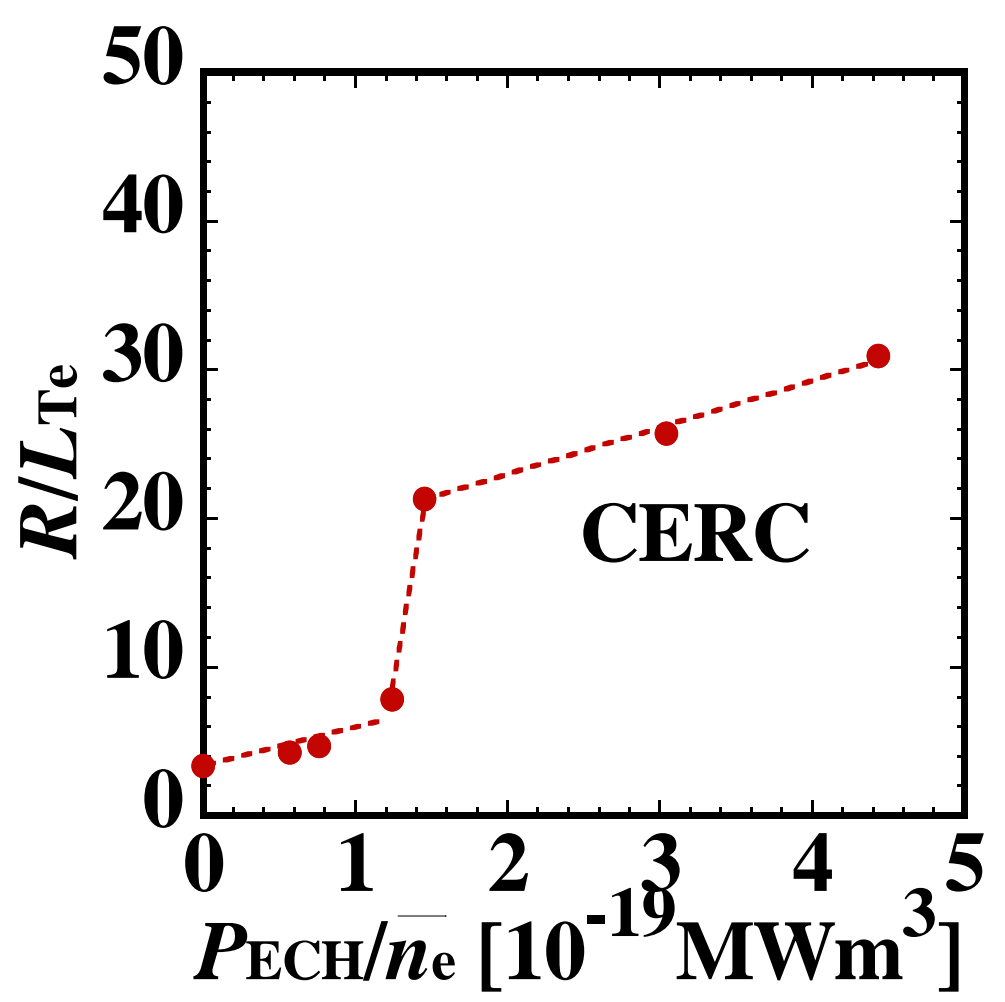

(Non-color on printed journal) 
Fig.6 NF/241006/PAP Yokoyama et al.

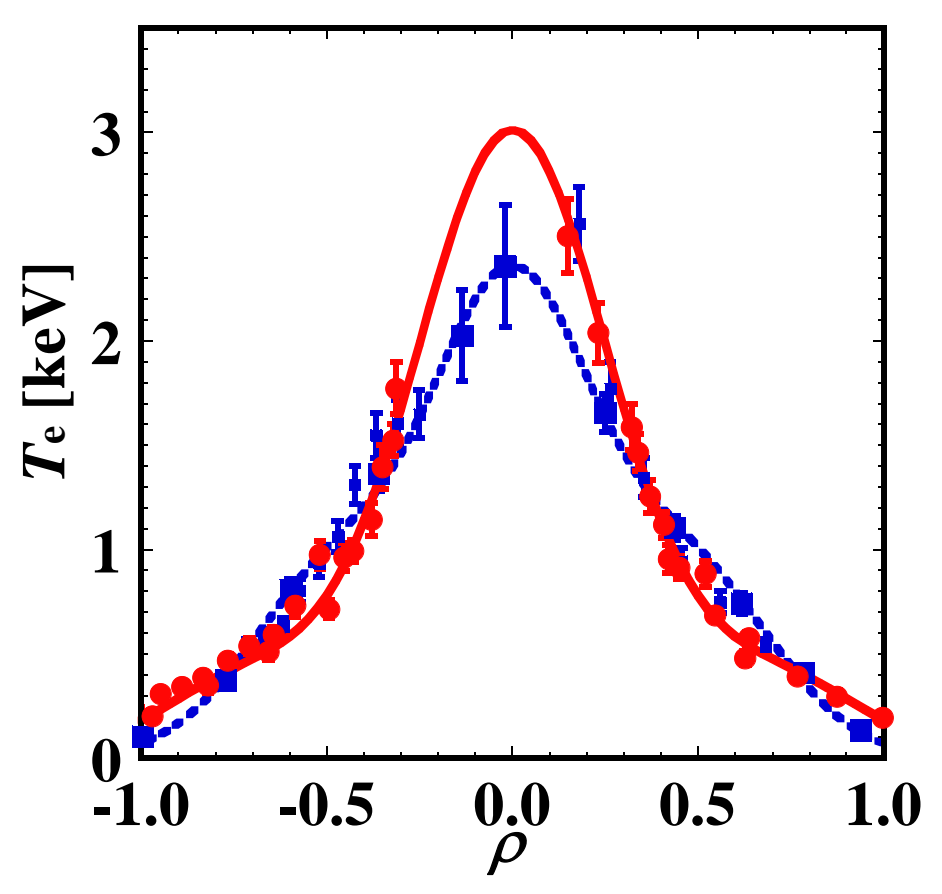

(Non-color on printed journal) 


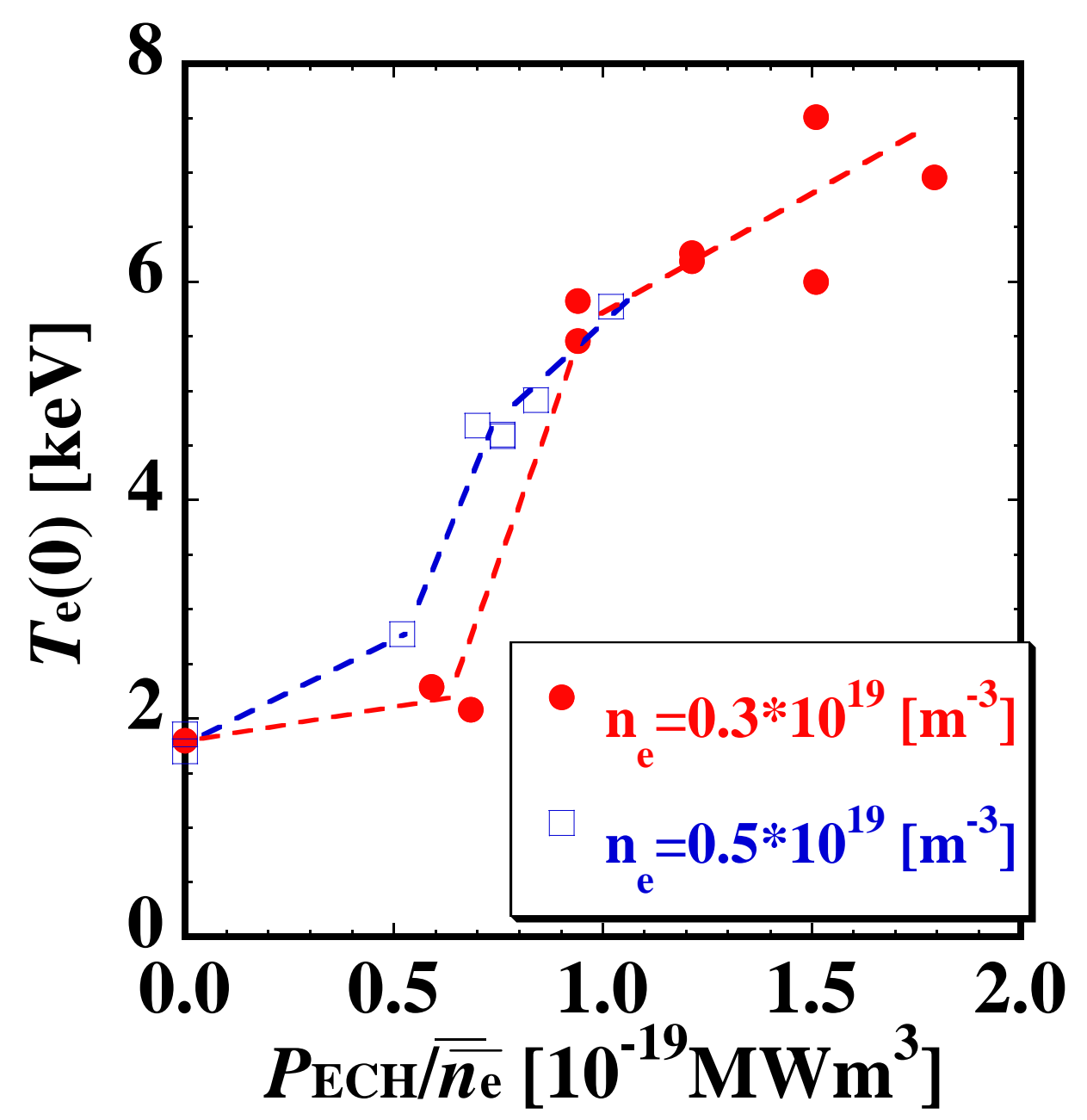

(Non-color on printed journal) 


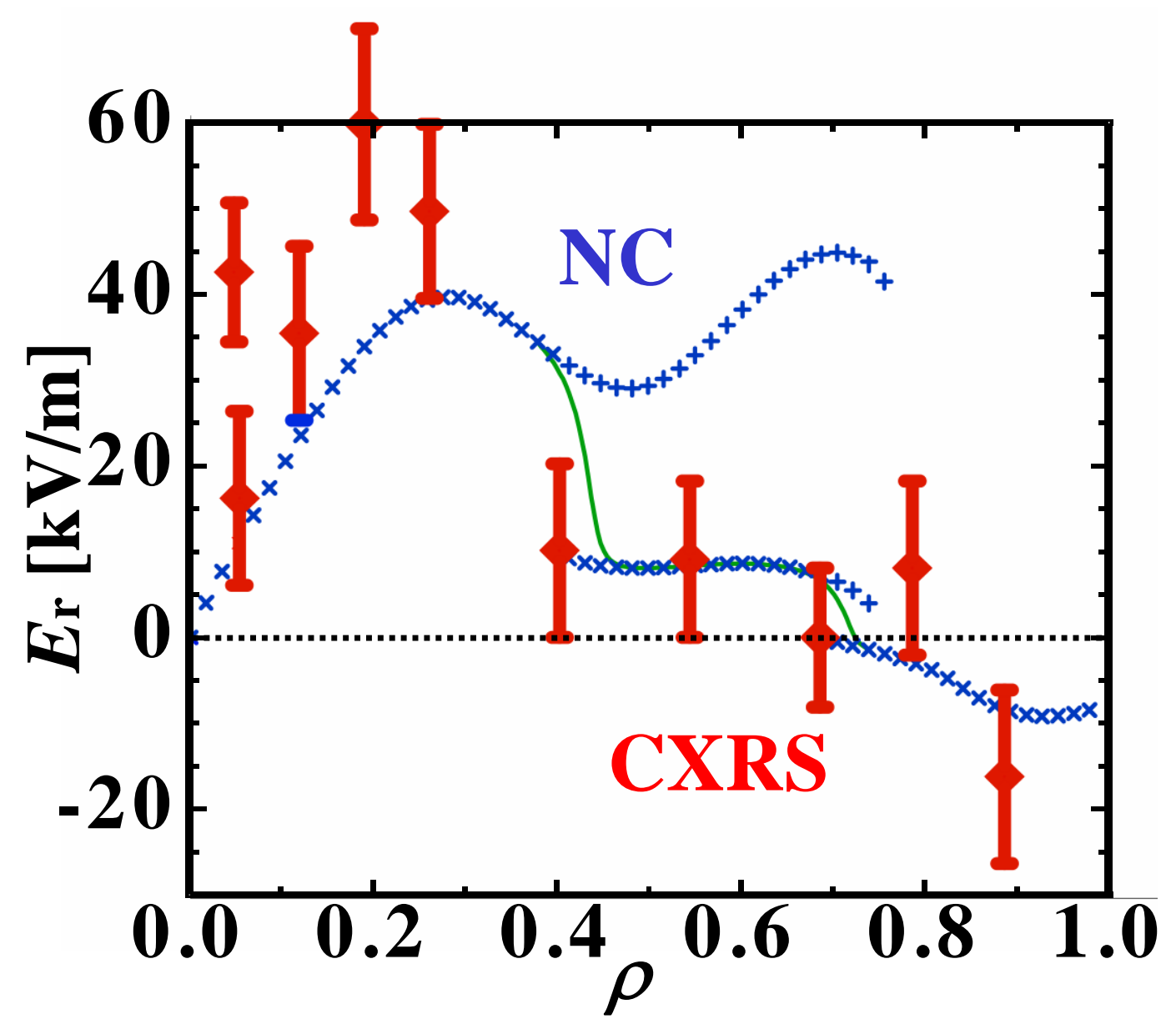

(Non-color on printed journal) 


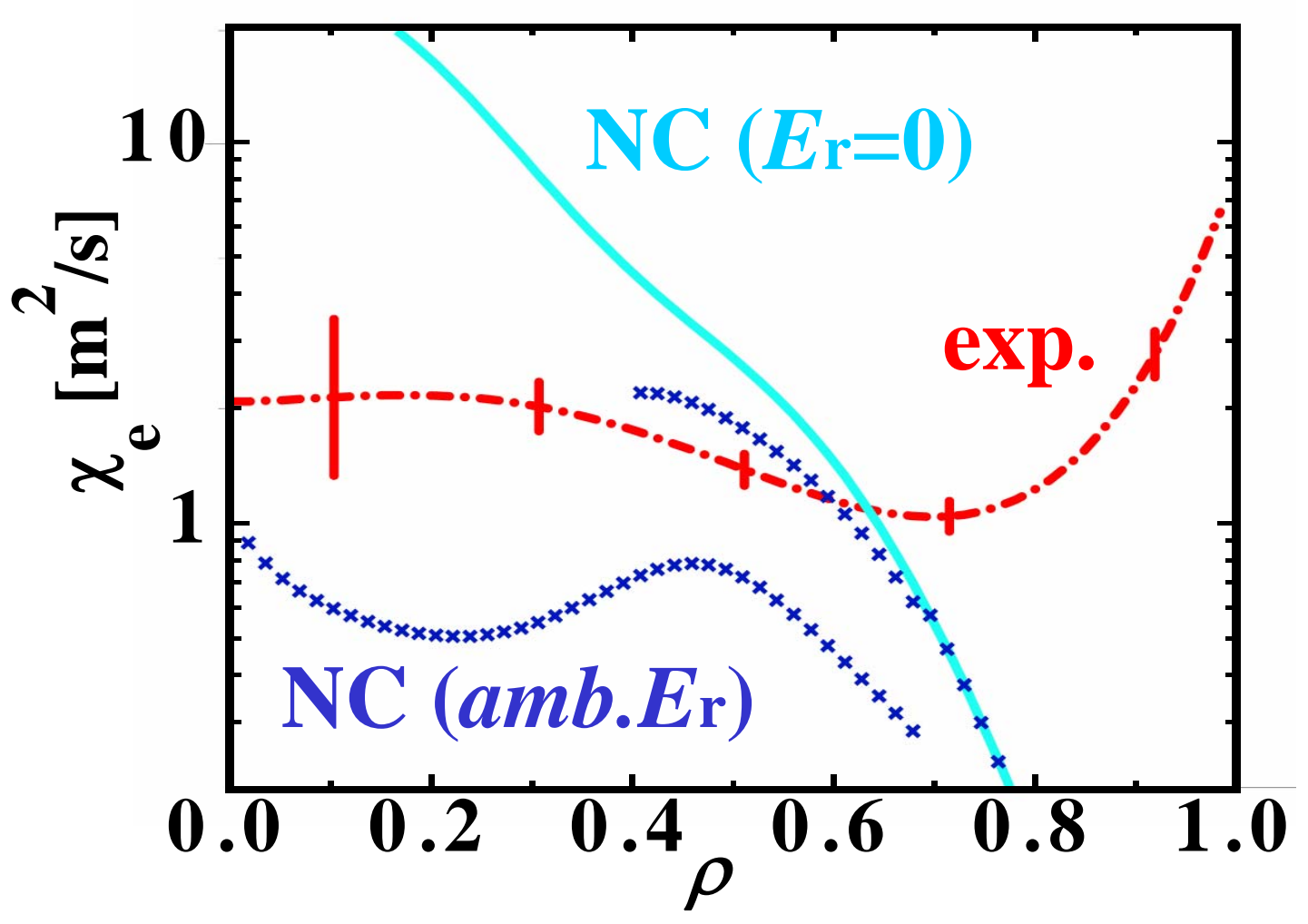

(Non-color on printed journal) 